\title{
CAPÍTULO 01: EFEITOS INIBITÓRIOS DO EXTRATO AQUOSO DO CHÁ ROXO (Camellia sinensis) SOBRE A $\alpha$-AMILASE E A LIPASE PANCREÁTICAS
}

\section{CAPÍTULO 01: EFECTOS INHIBIDORES DEL EXTRACTO DE TÉ PÚRPURA (Camellia sinensis) ACUOSO SOBRE $\alpha$-AMILASA Y LIPASA PANCREÁTICAS}

\section{CHAPTER 01: INHIBITORY EFFECTS OF AQUEOUS PURPLE TEA (Camellia sinensis) EXTRACT ON PANCREATIC $\alpha$-AMYLASE AND LIPASE}

\author{
Tamires Barlati Vieira da Silva ${ }^{1}$; Isabella Cristina de Angelo Delmiro ${ }^{2}$; Letícia Camargo Hass ${ }^{3}$; Adelar Bracht $^{4}$; \\ Rosane Marina Peralta ${ }^{5}$.
}

DOI: https://doi.org/10.31692/978-65-88970-17-1.1-15

\begin{abstract}
RESUMO
Um dos tratamentos mais comuns da obesidade e do diabetes envolve a prescrição de inibidores das amilases e lipases pancreáticas, que reduzem a hidrólise de triglicerídeos e carboidratos complexos com consequente redução de ácidos graxos e glicose disponíveis para absorção pelas células intestinais. Extratos de plantas são possíveis substitutos para inibidores convencionais de amilase e lipase. As folhas de chá (Camellia sinensis) são uma valiosa fonte de produtos metabólicos secundários, incluindo flavonóides, alcalóides, polissacarídeos entre outras. Entre esses metabólitos secundários, os flavonóides (que compreendem polifenóis, flavonas, flavanonóis e antocianinas) são micronutrientes com vários efeitos benéficos para a saúde humana, exercendo papéis protetores contra doenças cardiovasculares, doenças inflamatórias e câncer. O chá roxo é uma variedade rara de chá cultivada no Quênia. É elaborado a partir de uma nova variedade mestiça da folha do chá comum, Camellia sinensis, da qual derivam os chás preto, branco e verde. Esta nova variedade da planta clássica de chá foi desenvolvida apenas recentemente e é exclusiva do Quênia. Ao contrário das variedades tradicionais, até o momento, nenhum estudo foi realizado para avaliar uma provável ação inibitória do chá roxo sobre as enzimas digestivas. Assim, o objetivo desse estudo foi avaliar o potencial inibitório do chá roxo sobre as enzimas amilase e lipase pancreáticas. Através dos experimentos realizados, pode-se calcular um valor de $\mathrm{IC}_{50}$ (concentração do extrato de chá roxo que causou uma inibição de $50 \%$ da atividade da enzima) de $1,25 \mathrm{mg} / \mathrm{mL}$ e $0,17 \mathrm{mg} / \mathrm{mL}$ para a amilase e para a lipase, respectivamente. Os dados obtidos sugerem que o extrato de chá roxo, graças às suas atividades inibitórias sobre as enzimas digestivas possa ser um coadjuvante ou mesmo servir de base para um futuro fármaco útil no controle da obesidade e diabetes.
\end{abstract}

Palavras-chave:Chá roxo, inibição das enzimas digestivas, amilase, lipase

\section{RESUMEN}

Uno de los tratamientos más habituales para la obesidad y la diabetes consiste en la prescripción de inhibidores de la amilasa y lipasa pancreáticas, que reducen la hidrólisis de triglicéridos y carbohidratos complejos con la consiguiente reducción de ácidos grasos y glucosa disponibles para ser absorbidos por las células intestinales. Los extractos de plantas son posibles sustitutos de los inhibidores convencionales de amilasa y lipasa. Las hojas de té (Camellia sinensis) son una valiosa fuente de productos metabólicos secundarios, que incluyen flavonoides, alcaloides, polisacáridos, entre otros. Entre estos metabolitos secundarios, los flavonoides (que comprenden polifenoles, flavonas, flavanonoles y antocianinas) son micronutrientes con diversos efectos beneficiosos para la salud humana, que desempeñan funciones

\footnotetext{
${ }^{1}$ Programa de Pós-graduação em Ciência de Alimentos, Universidade Estadual de Maringá, tamiresbarlati93@gmail.com

${ }^{2}$ Graduação em Engenharia de Alimentos, bolsista PIBIC, Universidade Estadual de Maringá, belladeangelo97@gmail.com

${ }^{3}$ Graduação em Engenharia de Alimentos, bolsista PIBIC, Universidade Estadual de Maringá, leticiahass98@gmail.com

${ }^{4}$ Departamento de Bioquímica, Universidade Estadual de Maringá, adebracht@uol.com.br

${ }^{5}$ Departament de Bioquímica, Universidade Estadual de Maringá, rosanemperalta@gmail.com
} 


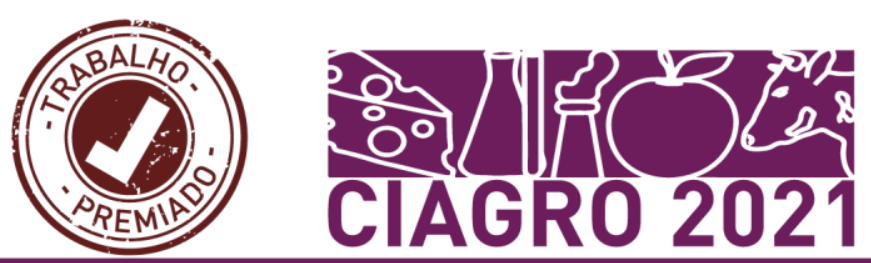

protectoras contra las enfermedades cardiovasculares, las enfermedades inflamatorias y el cáncer. El té púrpura es una variedad rara de té que se cultiva en Kenia. Se elabora a partir de una nueva variedad mestiza de la hoja de té común, Camellia sinensis, de la que se derivan los tés negro, blanco y verde. Esta nueva variedad de la clásica planta de té se desarrolló recientemente y es exclusiva de Kenia. A diferencia de las variedades tradicionales, hasta la fecha, no se ha realizado ningún estudio para evaluar una probable acción inhibidora del té púrpura sobre las enzimas digestivas. Por lo tanto, el objetivo de este estudio fue evaluar el potencial inhibidor del té púrpura sobre las enzimas amilasa y lipasa pancreáticas. Através de los experimentos realizados, se obtuvo un valor de $\mathrm{IC}_{50}$ (concentración de extracto de té púrpura que provocó una inhibición del $50 \%$ de la actividad enzimática) de $1,25 \mathrm{mg} / \mathrm{mL}$ y $0,17 \mathrm{mg} / \mathrm{mL}$ para amilasa y para lipasa, respectivamente. Los datos obtenidos sugieren que el extracto de té púrpura, gracias a sus actividades inhibidoras sobre las enzimas digestivas, puede ser un coadyuvante o incluso servir de base para un futuro fármaco útil en el control de la obesidad y la diabetes.

Palabras clave: Té púrpura, inhibición de enzimas digestivas, amilasa, lipasa

\begin{abstract}
One of the most common treatments for obesity and diabetes involves the prescription of inhibitors of pancreatic amylases and lipases, which reduce the hydrolysis of triglycerides and complex carbohydrates with a consequent reduction in fatty acids and glucose available for absorption by intestinal cells. Plant extracts are possible substitutes for conventional amylase and lipase inhibitors. Tea leaves (Camellia sinensis) are a valuable source of secondary metabolic products, including flavonoids, alkaloids, polysaccharides, among others. Among these secondary metabolites, flavonoids (which comprise polyphenols, flavones, flavanonols and anthocyanins) are micronutrients with several beneficial effects on human health, playing protective roles against cardiovascular diseases, inflammatory diseases and cancer. Purple tea is a rare variety of tea grown in Kenya. It is made from a new mestizo variety of the common tea leaf, Camellia sinensis, from which black, white and green teas are derived. This new variety of the classic tea plant was developed only recently and is unique to Kenya. Unlike traditional varieties, to date, no study has been carried out to assess a likely inhibitory action of purple tea on digestive enzymes. Thus, the aim of this study was to evaluate the inhibitory potential of purple tea on pancreatic amylase and lipase enzymes. Through the experiments carried out, an $\mathrm{IC}_{50}$ value (concentration of purple tea extract that caused a 50\% inhibition of the enzyme activity) of $1.25 \mathrm{mg} / \mathrm{mL}$ and $0.17 \mathrm{mg} / \mathrm{mL}$ for amylase and for lipase, respectively. The data obtained suggest that the purple tea extract, thanks to its inhibitory activities on digestive enzymes, may be an adjunct or even serve as a basis for a future drug useful in the control of obesity and diabetes.
\end{abstract}

Key words: Purple tea, inhibition of digestive enzymes, lipase, amylase.

\title{
INTRODUÇÃO
}

A obesidade e o diabetes são problemas de saúde pública mundial, com custos sociais incalculáveis (GAMBOA-GÓMEZ et al., 2015). Muitos medicamentos tem sido utilizados para controlar tanto a obesidade quanto o diabetes ao longo dos anos. Muitos destes medicamentos causam sérios efeitos adversos, como dor abdominal, flatulência, diarreia e diminuição da absorção de vitaminas (KANG; PARK, 2012). Por esta razão, compostos naturais têm sido explorados como potenciais ferramentas seguras no tratamento da obesidade e do diabetes. Alimentos ricos em compostos fenólicos da dieta têm atraído muito interesse devido às suas propriedades biológicas benéficas, que podem desempenhar um papel importante na manutenção da saúde humana. Diversos estudos demonstraram que extratos vegetais ricos em compostos fenólicos, como ácidos fenólicos, flavonoides e antocianinas, têm efeitos inibitórios 


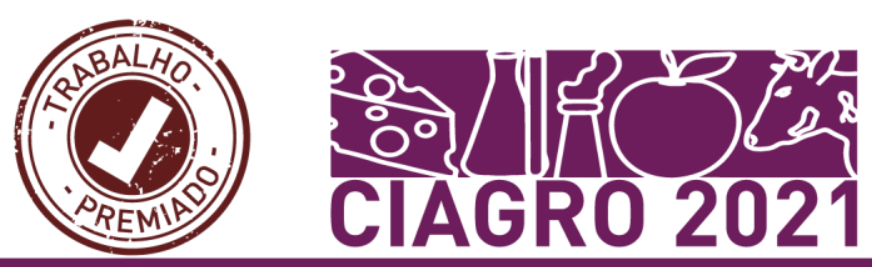

contra lipases e amilases e poderiam, em tese, serem utilizados para redução da absorção de lipídeos e carboidratos complexos, respectivamente (CHIOU et al., 2018).

Os chás provenientes das folhas de Camellia sinensis são ricos em compostos fenólicos e antioxidantes. O chá verde, obtido das folhas secas não fermentadas da planta é o mais conhecido deles, e muitos estudos associam o seu consumo a diversos benefícios à saúde como prevenção de ganho de peso corporal, controle e prevenção de diabetes, doenças cardiovasculares, câncer e outras doenças (YANG; WANG; SHERIDAN, 2018).

O chá roxo é obtido de uma nova variedade da planta Camellia sinensis desenvolvida no Quênia. A coloração do chá roxo é devida ao seu mais elevado teor em antocianinas, que podem estar associadas às suas múltiplas propriedades funcionais, incluindo propriedades antioxidantes, quimiopreventivas, antibacterianas, antiangiogênicas, anti-inflamatórias e antiateroscleróticas, podendo ainda ser utilizado como corante natural (KERIO et al., 2012). Até o momento, nenhum estudo foi realizado para avaliar uma provável ação inibitória do chá roxo sobre as enzimas digestivas o que poderia torná-lo um coadjuvante ou mesmo servir de base para um futuro fármaco útil no controle da obesidade e diabetes. Assim, o objetivo desse estudo foi avaliar o potencial inibitório do chá roxo (Camellia sinensis) sobre as enzimas amilase e lipase pancreáticas.

\section{REFERENCIAL TEÓRICO}

Nos últimos anos a obesidade e o diabetes estão cada vez mais comuns,tornando-se desafios clínicos e de saúde pública em todo o mundo. A urbanização, sedentarismo e ingestão excedente de energia e alimentos industrializados são algumas das causas do aumento de casos (KAUR; JAYASEKERA; MOUGHAN, 2014). Um dos tratamentos mais comuns da obesidade e do diabetes envolve a prescrição de inibidores enzimáticos, que reduzema absorção de ácidos graxos e glicose pelas células intestinais. Isso ocorre devido às enzimas digestivas amilases e lipases serem responsáveis pela hidrólise dos carboidratos complexos e lipídios da dieta (AWOSIKA; ALUKO, 2019).Alguns exemplos de fármacos utilizados nesta abordagem farmacológica são a acarbose, inibidor das amilases e orlistat, inibidor da lipase pancreática. Tais medicamentos provocam efeitos colaterais negativos como, desconforto gastrointestinal, flatulência, cólicas abdominais e diarréia (AWOSIKA; ALUKO, 2019; GUTIÉRREZGRIJALVA et al., 2019).

Acredita-se que diferentes moléculas encontradas em partes de plantas (folhas, frutos, cascas, etc) possam ser úteis na prevenção e no tratamento da obesidade e diabetes. Tal 


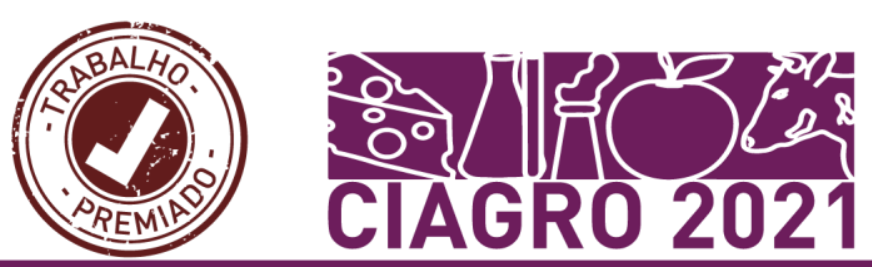

possibilidade, muito explorada nos últimos anos, demonstra grande potencial para o desenvolvimento de novos fármacos seguros e eficazes (ERCAN; EL, 2016; AWOSIKA; ALUKO, 2019). Os chás são ótimas alternativas no controleda obesidade e diabetes devido à ligação de seus polifenóis e da cafeína aos lipídios e proteínas (incluindo inibições das enzimas digestivas) resultando em uma menor absorção de macronutrientes (YANG et al., 2018).

As principais enzimas envolvidas na hidrólise de carboidratos e gorduras, são: alfaamilases e alfa-glicosidase e a lipase, respectivamente (ERCAN; EL, 2016). A alfa-amilase (EC 3.2.1.1) juntamente com a alfa-glicosidase (EC 3.2.1.20) são as principais enzimas envolvidas na hidrólise de polissacarídeos complexos. A alfa-amilase hidrolisa os polissacarídeos em oligossacarídeos e a alfa-glicosidase catalisa a etapa final da hidrólise liberando os monossacarídeos absorvíveis para entrar na corrente sanguínea (TRINH; STAERK; JÄGER, 2016). Potenciais inibidores das enzimas $\alpha$-amilase e $\alpha$-glucosidase podem ser usados clinicamente como agentes quimioterapêuticos para o tratamento do diabetes e da obesidade, pois os mesmos causam uma redução da absorção de glicose e impedem a elevação da glicemia pós-prandial (XU et al., 2018; HAMEED et al., 2019).

A lipase pancreática (EC 3.1.1.3) é uma enzima essencial para a digestão de gorduras alimentares no lúmen intestinal. Essa enzima é secretada pelo pâncreas e catalisa rapidamente a hidrólise do triglicerídeo (TAG) em um 2-monoglicerídeo e dois ácidos graxos livres (MUKHERJEE, 2003). Portanto, a inibição da lipase pancreática diminui os ácidos graxos livres e os monoacilgliceróis no lúmen intestinal, resultando em menor absorção dos TAG da dieta (SOSNOWSKA et al., 2015).

As folhas de chá (Camellia sinensis) são uma valiosa fonte de produtos metabólicos secundários, incluindo flavonóides, alcalóides, polissacarídeos entre outras. Entre esses metabólitos secundários, os flavonóides (que compreendem polifenóis, flavonas, flavanonóis e antocianinas) são micronutrientes com vários efeitos benéficos para a saúde humana, exercendo papéis protetores contra doenças cardiovasculares, doenças inflamatórias e câncer (SINGH; AKHTAR; HAQQI, 2010; NAMAL SENANAYAKE, 2013).

O chá roxo é uma variedade rara de chá cultivada no Quênia. É elaborado a partir de uma nova variedade mestiça da folha do chá comum, Camellia sinensis, da qual derivam os chás preto, branco e verde. Esta nova variedade da planta clássica de chá foi desenvolvida apenas recentemente e é exclusiva do Quênia. Comparado com o chá convencional, o chá roxo é rico em antocianinas que tem múltiplas propriedades funcionais, incluindo propriedades antioxidantes, quimiopreventivas, antibacterianas, antiangiogênicas, anti-inflamatórias e antiateroscleróticas, podendo ainda ser utilizado como corante natural (KERIO et al., 2012). Apesar 


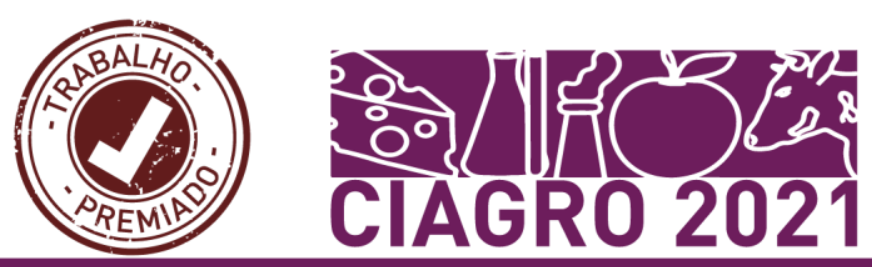

de a ação inibitória do chá verde sobre as amilases e lipases já terem sido relatadas, nenhum estudo foi realizado com o chá roxo, o que justifica este trabalho.

\section{METODOLOGIA}

A pesquisa realizada foi de natureza quantitativa experimental. Folhas do chá roxo do Quênia foram adquiridas da empresa em Justea.com, Vancouver, Canadá. Os extratos foram preparados de acordo com o protocolo descrito previamente (LOCHOCKA et al., 2015). As folhas de chá (100 g) foram moídas, fervidas em água destilada (1 L) e subsequentemente agitadas durante 15 minutos a $70{ }^{\circ} \mathrm{C}$ (repetido 3 vezes). Os extratos coletados foram filtrados, congelados, liofilizados e guardados a $-20^{\circ} \mathrm{C}$. A $\alpha$-amilase pancreática suína (tipo IV-B), lipase pancreática suína (tipo II), amido de batata e p-nitro-fenil-palmitato (substrato sintético da lipase) foram adquiridos da Sigma-Aldrich.

Para avaliação da inibição da amilase pancreática, medidas de velocidade de reação foramrealizadas a $37^{\circ} \mathrm{C}$ em tampão fosfato $20 \mathrm{mM}$, pH 6,9 com $6.7 \mathrm{mM}$ de $\mathrm{NaCl}$ (KATO et al., 2017). Amido de batata a diferentes concentrações foi utilizado como substrato. Experimentos foram conduzidos na ausência (controle) e na presença de duas diferentes concentrações do extrato de chá roxo. Os açúcares redutores produzidos a partir da hidrólise do amido foram quantificados pelo método do ácido 3,5 dinitrosalicílico (DNS), usando glicose como padrão (MILLER, 1959). O grupo aldeído do açúcar redutor converte 3,5 dinitrosalicilato em 3- amino 5-nitrosalicilato, forma reduzida do DNS. Este absorve luz em 540nm. A absorbância determinada é diretamente proporcional a quantidade de açúcar redutor.

Para avaliação da inibição da lipase pancreática, as velocidades de reação foram determinadas espectrofotometricamente a 410nm, usando p-nitrofenil-palmitato como substrato (OLIVEIRA et al., 2015). O substrato foi preparado em isopropanol e sonicado até completa solubilização. No momento do uso, a solução estoque foi diluída com isopropanol até a concentração de $0,5 \mathrm{mg} / \mathrm{mL}$. A lipase pancreática de porco foi solubilizada em tampão Tris$\mathrm{HCl}$ (pH 8.0) na concentração de $2 \mathrm{mg} / \mathrm{mL}$. Após a centrifugação da suspensão $(2.000 \mathrm{~g}, 15$ min.), o sobrenadante foi usado como fonte enzimática. O meio de reação que continha 100 mM tampão Tris- $\mathrm{HCl}(\mathrm{pH}$ 8.2) e diferentes concentrações de substrato $p$-nitrofenil palmitato foi incubado a $37^{\circ} \mathrm{C}$. Um volume de $0,1 \mathrm{~mL}$ da lipase foi adicionado e a reação foi permitida ocorrer por 10 minutos na mesma temperatura. A reação foi interrompida por fervura. Após centrifugação, a absorbância do sobrenadante, que contém o íon $p$-nitrofenolato liberado do substrato $p$-nitrofenil-palmitato, foi lida a $410 \mathrm{~nm}$ contra uma solução de branco contendo 


\section{enzima desnaturada. Para avaliar a inibição do extrato de chá roxo, a enzima foi previamente}

incubada por 10 min. com duas diferentes concentrações do extrato e em seguida, a reação foi desenvolvida como descrito para o controle.

O software Scientist da MicroMath Scientific Software (Salt Lake City, UT) foi usado para ajustar as equações de velocidade às velocidades iniciais experimentais através de um ajuste iterativo não-linear de mínimos quadrados. A decisão sobre o modelo cinético mais adequado (equação) foi baseada no critério de seleção modelar (MSC, model selection criterium) e nos desvios padrões dos parâmetros otimizados. O MSC, que corresponde ao critério de informação normalizado de Akaike, é definido como (AKAIKE, 1974):

$$
\mathrm{MSC}=\ln \left[\frac{\sum_{\mathrm{i}=1}^{\mathrm{n}} \mathrm{w}_{\mathrm{i}}\left(\mathrm{Y}_{\mathrm{obs}}-\overline{\mathrm{Y}}_{\mathrm{obs}}\right)^{2}}{\sum_{\mathrm{i}=1}^{\mathrm{n}} \mathrm{w}_{\mathrm{i}}\left(\mathrm{Y}_{\mathrm{obs} \mathrm{i}_{1}}-\mathrm{Y}_{\mathrm{cal}}\right)^{2}}\right]-\frac{2 \mathrm{p}}{\mathrm{n}}
$$

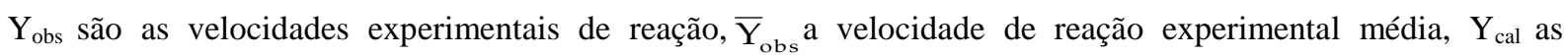
velocidades de reação calculadas, w o peso de cada ponto experimental, n o número de observações, e p o número de parâmetros da equação.

No presente trabalho, o modelo com o maior valor de MSC foi considerado o mais apropriado, desde que os parâmetros estimados tenham apresentado sinal positivo. Quando os valores de MSC diferiram em menos de 5\%, o modelo com os menores desvios padrões dos parâmetros estimados foi considerado como o mais apropriado.

\section{RESULTADOS E DISCUSSÃO}

Para investigar a influência da concentração do substrato na ação inibitória do extrato de chá roxo, as velocidades iniciais de reação foram medidas variando simultaneamente as concentrações do substrato e do inibidor (extrato de chá roxo). Os resultados estão mostrados na Figura 1. 


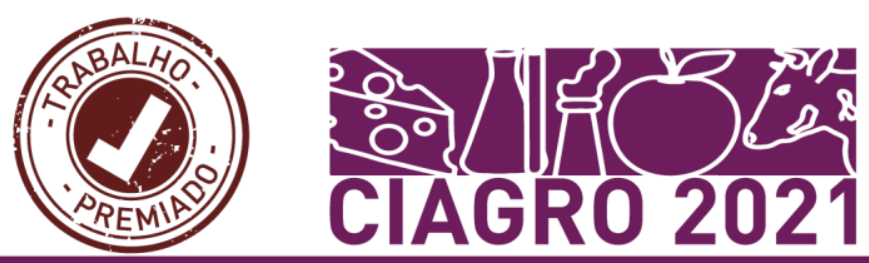

Figura 1. Efeitos do extrato do chá roxo sobre as velocidades iniciais de hidrólise do amido catalisada pela $\alpha$ amilase pancreática suína a diferentes concentrações de amido e do extrato de chá roxo. Cada ponto experimental é a média de três determinações. As linhas que percorrem os pontos experimentais foram calculadas usando os parâmetros otimizados obtidos pelo ajuste da equação (2) aos dados experimentais por meio de um procedimento não linear de mínimos quadrados.

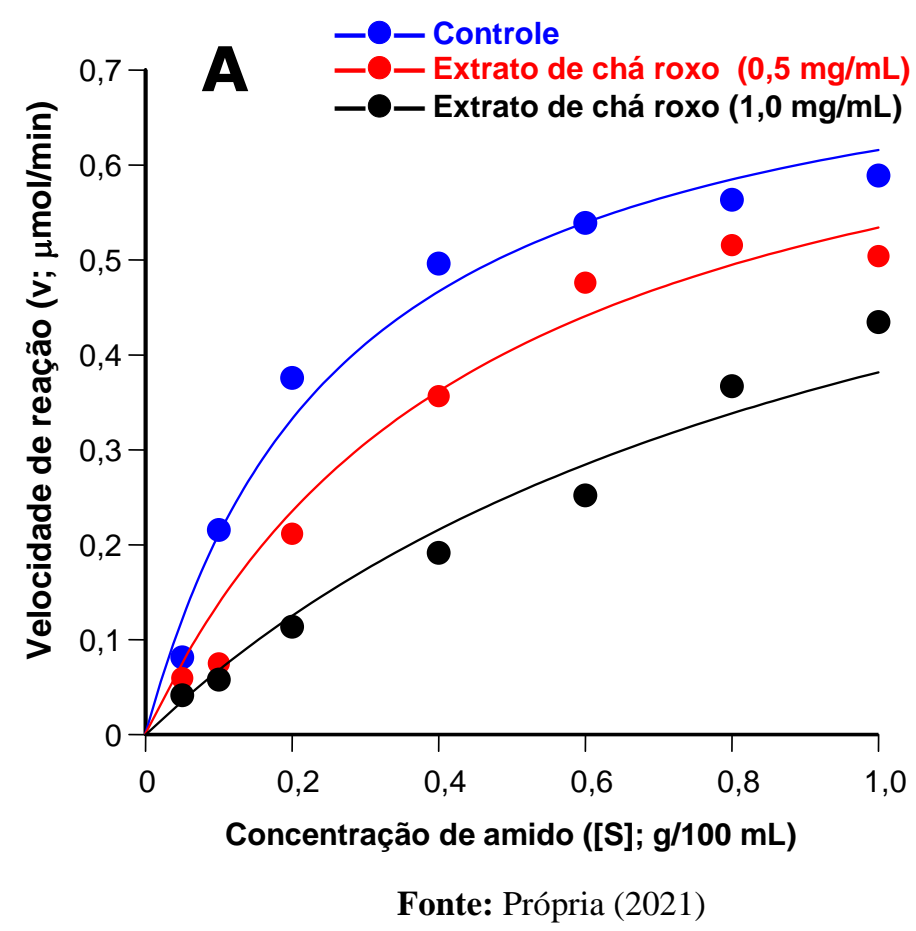

A dependência da atividade da $\alpha$-amilase pancreática da concentração do substrato mostra a curva de saturação usual, conforme relatado previamente (DA SILVA et al., 2014). As duas curvas de saturação obtidas com as duas concentrações de extrato de chá roxo correm abaixo da curva-controle, denotando inibição. Foram feitas tentativas para ajustar várias equações cinéticas que descrevem a inibição não competitiva, mista ou competitiva para todo o conjunto de dados por meio de um procedimento de mínimos quadrados não linear. Com base nos critérios estatísticos delineados na seção Materiais e Métodos, a equação que deu o melhor ajuste aos dados experimentais foi a seguinte:

$$
\mathrm{v}=\frac{\mathrm{V}_{\max }[\mathrm{S}]}{\mathrm{K}_{\mathrm{M}}\left(1+\frac{[\mathrm{I}]^{2}}{\left(\overline{\mathrm{K}}_{\mathrm{i} 1}\right)^{2}}\right)+[\mathrm{S}]}
$$

Na equação acima, $\mathrm{K}_{\mathrm{M}}$ é a constante de Michaelis, $\mathrm{V}_{\max }$ corresponde à velocidade máxima e $\overline{\mathrm{K}}_{\mathrm{i} 1}$ é a constante média de inibição.

A equação descreve uma inibição competitiva parabólica em que IEI é o complexo enzima-inibidor predominante (DA SILVA et al., 2014). Os valores dos parâmetros otimizados e indicadores de qualidade de ajuste são: $\mathrm{K}_{\mathrm{M}}, 0,270 \pm 0,047 \mathrm{~g} / 100 \mathrm{~mL} ; \mathrm{V}_{\text {máx }}, 0,782 \pm 0,046$ 
$\mu \mathrm{mol} / \mathrm{min} ; \bar{K}_{i 1}, 0,589 \pm 0,242 \mathrm{mg} / \mathrm{mL}$; soma dos quadrados dos desvios, 0,0185; MSC, 3,402;

correlação, 0,98. A formação dos complexos IES ou IESI não pôde ser detectada com os dados mostrados na Figura 1 porque todas as tentativas de ajustar as equações de velocidade pertinentes produziram valores tendendo ao infinito para as constantes de inibição correspondentes.

A ampla predominância do componente competitivo na inibição causada pelo extrato de chá roxo implica em que o grau de inibição deverá variar com a concentração do substrato. Isso pode ser demonstrado usando os parâmetros cinéticos otimizados para calcular os valores de $\mathrm{IC}_{50}$ (concentração de inibidor que causa $50 \%$ de inibição) em função da concentração de substrato (amido) por meio da equação:

$$
\mathrm{IC}_{50}=\left[\left(\overline{\mathrm{K}}_{\mathrm{i} 1}\right)^{2}\left(1+\frac{[\mathrm{S}]}{\mathrm{K}_{\mathrm{M}}}\right)\right]^{1 / 2}
$$

Os resultados desses cálculos são mostrados na Figura 2 e revelam que em altas concentrações de amido o valor de $\mathrm{IC}_{50}$ é $1,25 \mathrm{mg} / \mathrm{mL}$, mas se aproxima do valor da constante de inibição do composto $\left(\bar{K}_{i 1}=0,59 \mathrm{mg} / \mathrm{mL}\right)$, quando a concentração de substrato é diminuída. Em outras palavras, o grau de inibição aumenta à medida que a concentração de substrato diminui.

Quando comparamos os valores obtidos deste estudo com o chá roxo com trabalhos já realizados com o chá verde ( $\mathrm{IC}_{50}$ de 4,02 mg/mL, GAO et al., 2013), podemos notar que o chá roxo exerce um efeito inibidor maior. Alguns estudos consideram as antocianinas como potenciais inibidoras da $\alpha$-amilase, além de melhorar a sensibilidade das células à insulina (GUO et al., 2008; HOMOKI et al., 2016; CHEN et al., 2019). Também já foi sugerido a existência de uma correlação significativa entre as antocianinas do chá roxo e a sua atividade antioxidante (LV et al., 2015). 
Figura 2. Valores calculados de $\mathrm{IC}_{50}$ do extrato de chá roxo em função da concentração do substrato (amido). Os valores de $\mathrm{IC}_{50}$ foram computados através da equação (3), usando os parâmetros otimizados.

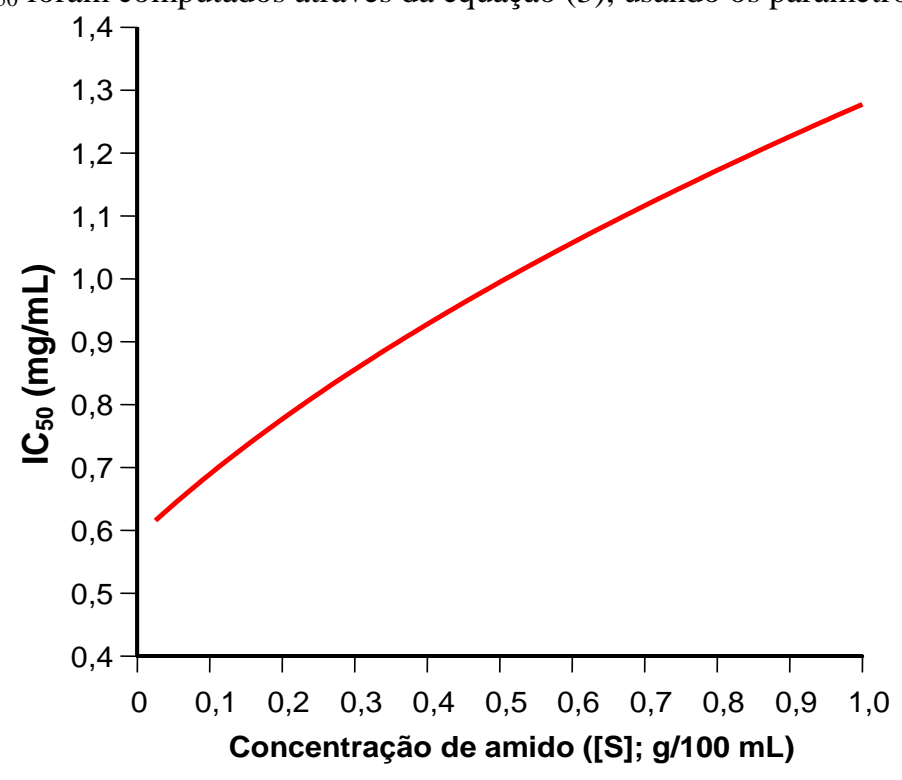

Fonte: Própria (2021)

A atividade inibidora do extrato de chá roxo sobre a lipase pancreática está ilustrada pela Figura 3. De forma análoga ao que foi feito com a $\alpha$-amilase, as velocidades iniciais de reação foram medidas variando simultaneamente as concentrações do substrato ( $p$-nitrofenilpalmitato) e do extrato de chá roxo. A curva de saturação da enzima para com o substrato utilizado apresentou o já relatado indício de uma inibição por substrato (ALVES CASTILHO et al., 2021). Cada uma das duas concentrações do extrato de chá roxo utilizadas comprimiu a curva de saturação, sendo que este efeito intensificou-se quando a concentração foi aumentada de 50 para $200 \mu \mathrm{g} / \mathrm{mL}$.

Para extrair das curvas de concentração parâmetros quantitativos, foram feitos tentativas de ajuste a diferentes modelos cinéticos, conforme delineado na seção de materiais e métodos. A equação que melhor descreve o conjunto de dados da Figura 3 é uma função que incluir a inibição por substrato e a formação dos complexos IEI e IESI:

$$
\mathrm{v}=\frac{\mathrm{V}_{\max }[\mathrm{S}]}{\mathrm{K}_{\mathrm{M}}\left(1+\frac{[\mathrm{I}]^{2}}{\left(\overline{\mathrm{K}}_{\mathrm{i} 1}\right)^{2}}\right)+[\mathrm{S}]\left(1+\frac{[\mathrm{I}]^{2}}{\left(\overline{\mathrm{K}}_{\mathrm{i} 2}\right)^{2}}\right)\left(1+\frac{[\mathrm{S}]}{\left(\mathrm{K}_{\mathrm{IS}}\right)}\right)}
$$




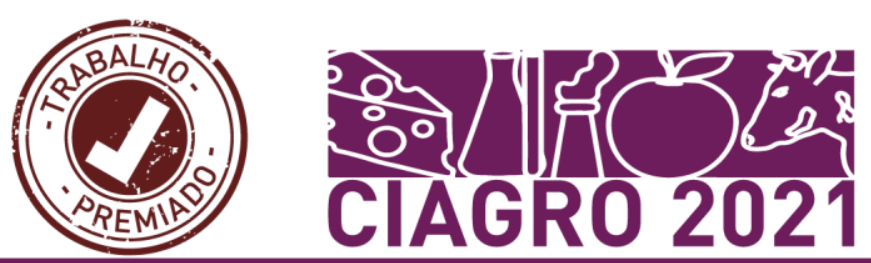

Figura 3. Efeitos do extrato do chá roxo sobre as velocidades iniciais de hidrólise do $p$-fenil palmitato catalisada pela lipase pancreática suína a diferentes concentrações do substrato. Cada ponto experimental é a média de duas determinações. As linhas que percorrem os pontos experimentais foram calculadas usando os parâmetros otimizados obtidos pelo ajuste da equação (4) aos dados experimentais por meio de um procedimento não linear de mínimos quadrados.

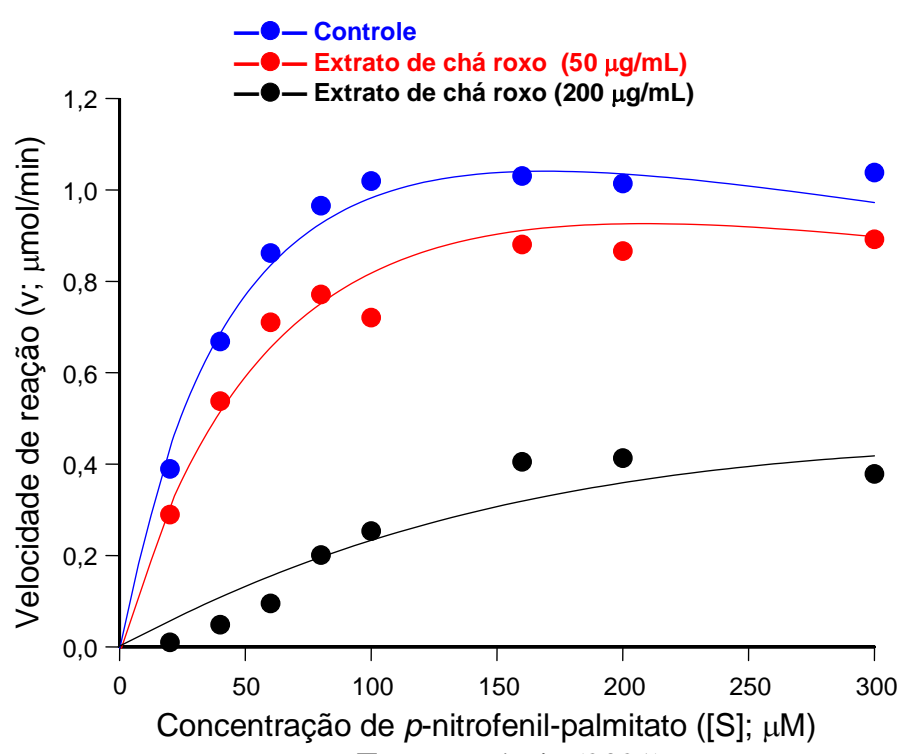

Fonte: Própria (2021)

$\mathrm{K}_{\mathrm{IS}}$ é a constante de inibição pelo substrato, $\overline{\mathrm{K}}_{\mathrm{i} 1}$ reflete uma média das constantes de dissociação do complexo IEI e $\overline{\mathbf{K}}_{\mathrm{i} 2}$ representa a média das constantes de dissociação do complexo IESI. A Figura 3 permite comparar as curvas experimentais (pontos experimentais) com as curvas calculadas utilizando a equação (4) e os parâmetros otimizados. Embora haja uma certa dispersão dos dados, as curvas calculadas não mostram nenhum desvio sistemático das curvas experimentais. É preciso notar que o valor de $\overline{\mathrm{K}}_{\mathrm{i} 2}$ é bem maior do que $\overline{\mathrm{K}}_{\mathrm{i} 1}$, o que denota que o complexo IEI forma-se mais facilmente do que o complexo IESI. O componente competitivo, portanto, é mais acentuado que o componente não-competitivo. Mas foi possível detectá-lo, ao contrário do que ocorreu com a inibição da $\alpha$-amilase IESI não pôde ser detectada. Os valores dos parâmetros otimizados e indicadores de qualidade de ajuste são: $\mathrm{K}_{\mathrm{M}}$, $61,17 \pm 18,59 \mu \mathrm{M} ; \mathrm{V}_{\text {máx }}, 1,79 \pm 0,30 \mu \mathrm{mol} / \mathrm{min} ; \mathrm{K}_{\mathrm{IS}}=468,65 \pm 215,62 ; \bar{K}_{i 1}, 67,39 \pm 33,0 \mu \mathrm{g} / \mathrm{mL}$; $\overline{\mathrm{K}}_{\mathrm{i} 2}, 317,31 \pm 324,86 \mu \mathrm{g} / \mathrm{mL}$; soma dos quadrados dos desvios, 0,0512; MSC, 3,54; correlação, 0,99 .

A exemplo do que foi feito com a $\alpha$-amilase é de interesse verificar também para a lipase como se comporta o $\mathrm{IC}_{50}$ em função da concentração do substrato. A equação correspondente será mais complexa, devido ao fato de que a equação de velocidade também é mais complexa. A seguinte equação é válida: 


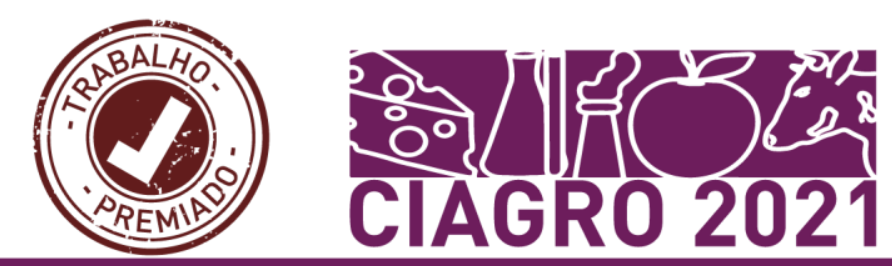

$$
\mathrm{IC}_{50}=\left[\frac{\mathrm{K}_{\mathrm{M}}+[\mathrm{S}]+\frac{[\mathrm{S}]^{2}}{\mathrm{~K}_{\mathrm{IS}}}}{\frac{\mathrm{K}_{\mathrm{M}}}{\left(\overline{\mathrm{K}}_{\mathrm{i} 1}\right)^{2}}+\frac{[\mathrm{S}]}{\left(\overline{\mathrm{K}}_{\mathrm{i} 2}\right)^{2}}+\frac{[\mathrm{S}]^{2}}{\mathrm{~K}_{\mathrm{IS}}\left(\overline{\mathrm{K}}_{\mathrm{i} 2}\right)^{2}}}\right]^{1 / 2}
$$

A equação (5) converge para a equação (3), no entanto, se não houver inibição por substrato $\left(\mathrm{K}_{\mathrm{IS}} \rightarrow \infty\right)$ e não houver a formação do complexo IESI $\left(\overline{\mathrm{K}}_{\mathrm{i} 2} \rightarrow \infty\right)$. No gráfico da Figura 4 foram representados os valores calculados de $\mathrm{IC}_{50}$ em função da concentração do substrato utilizando a equação (5) e os valores otimizados dos parâmetros cinéticos. É bem nítido o fato de que o valor do $\mathrm{IC}_{50}$ aumenta com incrementos na concentração do substrato, o que se deve em boa parte ao fato de que o componente competitivo predomina amplamente sobre o componente não-competitivo. Note-se que quando $[\mathrm{S}]$ tende a zero o valor do $\mathrm{IC}_{50}$ tende a um valor limite que corresponde ao valor de $\overline{\mathrm{K}}_{\mathrm{il}}(67,39 \mu \mathrm{g} / \mathrm{mL})$, enquanto o $\mathrm{IC}_{50}$ é $170 \mu \mathrm{g} / \mathrm{mL}$ para concentração saturante de substrato

Figura 4. Valores calculados de $\mathrm{IC}_{50}$ para a inibição da lipela pelo extrato de chá roxo em função da concentração do substrato ( $p$-nitrofenil-palmitato). Os valores de $\mathrm{IC}_{50}$ foram computados através da equação (5), usando os parâmetros otimizados.

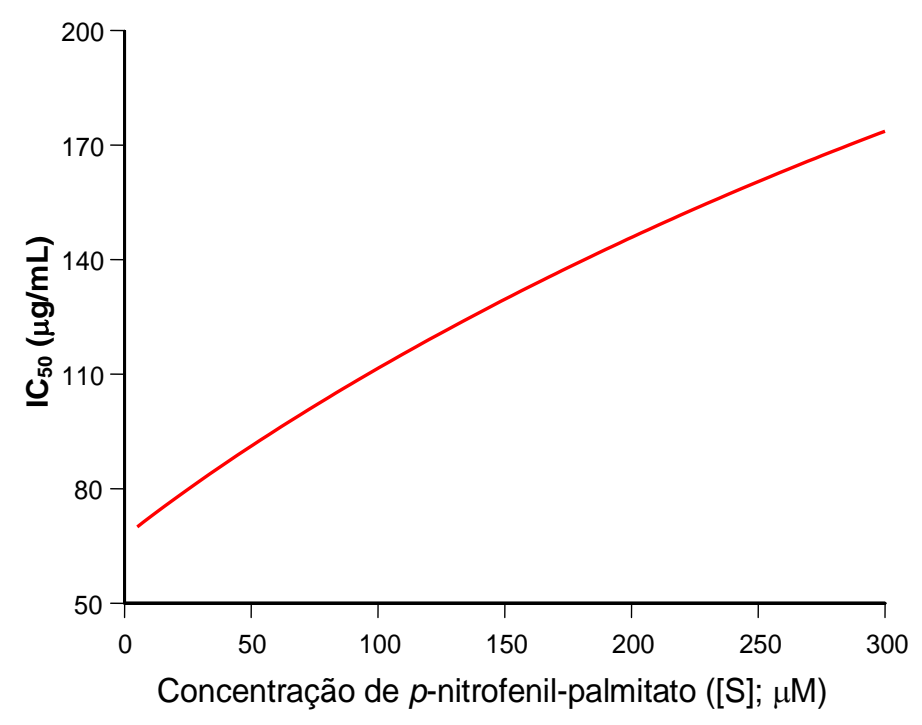

Fonte: Própria (2021)

Agora que os dados cinéticos para a lipase e a $\alpha$-amilase estão disponíveis pode-se comparar as ações inibidoras do extrato do chá roxo sobre as duas enzimas. Os componentes do chá roxo formam o complexo IEI com as duas enzimas. No caso da $\alpha$-amilase, a constante 


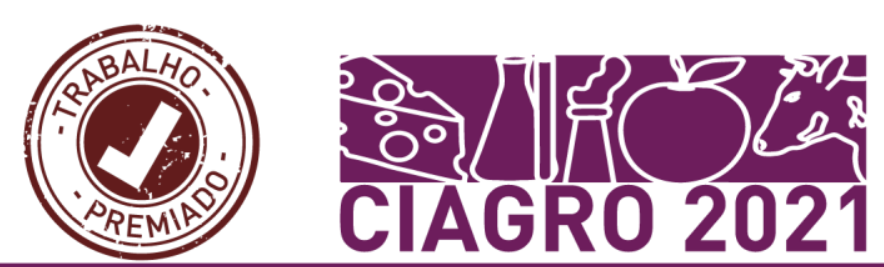

de dissociação correspondente $\left(\overline{\mathrm{K}}_{\mathrm{i} 1}\right)$ foi igual a $0,59 \mathrm{mg} / \mathrm{mL}$ enquanto que para a lipase o valor foi $0,067 \mathrm{mg} / \mathrm{mL}$. Portanto, os inibidores do chá roxo ligam-se 8,8 vezes mais fortemente à lipase do que à $\alpha$-amilase. Isto reflete-se também nos valores de $\mathrm{IC}_{50}$, que são bem menores no caso da lipase em comparação com os valores encontrados para a $\alpha$-amilase. A previsão que pode ser feita em relação aos possíveis efeitos dos extratos do chá roxo sobre a absorção (digestão) do amido e dos triglicerídeos, é que a ação sobre esta última deverá ser bem mais potente. Ação bem mais potente, por sua vez, significa doses menores. A diferença de 8,8 vezes na potência inibidora permite vislumbrar que seja possível inibir fortemente a absorção de gorduras com uma determinada dose a par de uma absorção pequena ou moderada da absorção do amido.

Vários estudos tem sugerido o uso de extratos naturais que possuem alto potencial para inibir a atividade da lipase pancreática, e minimizar os efeitos colaterais normalmente provocados por fármacos usuais (SEYEDAN et al., 2015; VIJAYARAJ; NAKAGAWA; YAMAKI, 2019). Quando se trata da relação dos chás provenientes da Camellia sinensis alguns estudos também sugerem seu potencial uso como inibidores da lipase. Os chás preto, verde e branco e seus polifenólicos já tiveram suas atividades inibidoras de lipases comparadas (HE; LV; YAO, 2007; GONDOIN et al., 2010). Em um estudo utilizando diferentes plantas mediterrâneas, os autores avaliaram a atividade inibitória da lipase pancreática in vitro de extratos liofilizados e correlacionaram esta inibição ao teor em antocianinas dos materiais (FABRONI et al., 2016). Mais recentemente, Castilho et al (2021), avaliaram o efeito inibitório da casca jabuticaba sobre a amilase e lipase pancreáticas. Entretanto neste estudo, os efeitos inibitórios não puderam ser correlacionados com os teores de cianidina-3-O-glucosídeo composto presente em maior quantidade na casca.

\section{CONSIDERAÇÕES FINAIS}

O extrato de chá roxo demonstrou ser inibidor das enzimas digestivas, $\alpha$-amilase e lipase pancreáticas. $\mathrm{O}$ efeito inibidor foi muito mais potente para a lipase pancreática. Os dados obtidos sugerem que o extrato de chá roxo pode ser útil no tratamento da obesidade e da diabetes. Futuros estudos usando modelos animais e humanos são necessários para confirmar os resultados obtidos a partir desta inibição in vitro. 


\section{REFERÊNCIAS}

AKAIKE, H. A new look at statistical model identification. IEEE Transactions Automatic Control v. 19, p. 716-723, 1973.AKAIKE, H. A new look at the statistical model identification. IEEE Transactions on Automatic Control, v. 19, n. 6, p. 716-723, dez. 1974. Disponível em: $<$ http://ieeexplore.ieee.org/document/1100705/>.

ALVES CASTILHO, P. et al. Effects of a Myrciaria jaboticaba peel extract on starch and triglyceride absorption and the role of cyanidin-3- O -glucoside. Food \& Function, v. 12, n. 6, p. 2644-2659, 2021. Disponível em: 〈http://xlink.rsc.org/?DOI=D0FO02927K〉.

AWOSIKA, T. O.; ALUKO, R. E. Inhibition of the in vitro activities of $\alpha$-amylase, $\alpha$ glucosidase and pancreatic lipase by yellow field pea ( Pisum sativum L.) protein hydrolysates. International Journal of Food Science \& Technology, v. 54, n. 6, p. 2021-2034, 4 jun. 2019. Disponível em: <https://onlinelibrary.wiley.com/doi/abs/10.1111/ijfs.14087>.

CHEN, X. et al. Wheat gluten protein inhibits $\alpha$-amylase activity more strongly than a soy protein isolate based on kinetic analysis. International Journal of Biological Macromolecules, v. 129, p. 433-441, 2019. Disponível em: <https://doi.org/10.1016/j.ijbiomac.2019.01.215>.

CHIOU, S. et al. In vitro inhibition of lipase, $\alpha$-amylase, $\alpha$-glucosidase, and angiotensinconverting enzyme by defatted rice bran extracts of red-pericarp rice mutant. Cereal Chemistry, v. 95, n. 1, p. 167-176, 25 jan. 2018. Disponível em: $<$ https://onlinelibrary.wiley.com/doi/abs/10.1002/cche.10025>.

DA SILVA, S. M. et al. Inhibition of salivary and pancreatic $\alpha$-amylases by a pinhão coat (Araucaria angustifolia) extract rich in condensed tannin. Food Research International, v. 56, p. 1-8, fev. 2014. Disponível em: <http://dx.doi.org/10.1016/j.foodres.2013.12.004〉.

ERCAN, P.; EL, S. N. Inhibitory effects of chickpea and Tribulus terrestris on lipase, $\alpha$-amylase and $\alpha$-glucosidase. Food Chemistry, v. 205, p. 163-169, ago. 2016. Disponível em: $<$ https://linkinghub.elsevier.com/retrieve/pii/S0308814616303545>.

FABRONI, S. et al. Screening of the anthocyanin profile and in vitro pancreatic lipase inhibition by anthocyanin-containing extracts of fruits, vegetables, legumes and cereals. Journal of the Science of Food and Agriculture, v. 96, n. 14, p. 4713-4723, nov. 2016. Disponível em: <http://doi.wiley.com/10.1002/jsfa.7708>.

GAMBOA-GÓMEZ, C. I. et al. Review article: PLANTS WITH POTENTIAL USE ON OBESITY AND ITS COMPLICATIONS. EXCLI Journal, v. 14, p. 809-831, 2015.

GAO, J. et al. Combined effects of green tea extracts, green tea polyphenols or epigallocatechin gallate with acarbose on inhibition against $\alpha$-amylase and $\alpha$-glucosidase in vitro. Molecules, v. 18, n. 9, p. 11614-11623, 2013.

GONDOIN, A. et al. White and green tea polyphenols inhibit pancreatic lipase in vitro. Food 


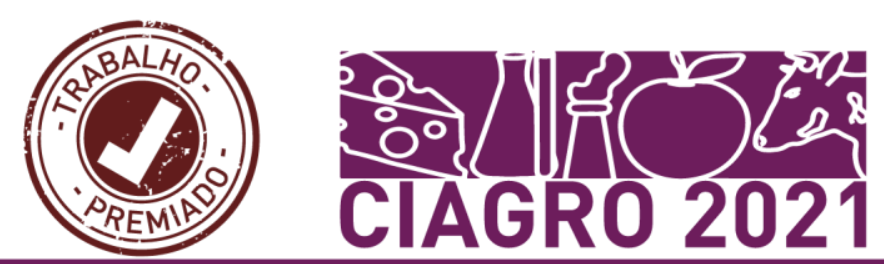

Research International, v. 43, n. 5, p. 1537-1544, 2010. Disponível em: <http://dx.doi.org/10.1016/j.foodres.2010.04.029>.

GUO, $\mathrm{H}$. et al. Cyanidin 3-glucoside protects 3T3-L1 adipocytes against $\mathrm{H} 2 \mathrm{O} 2-$ or TNF- $\alpha$ induced insulin resistance by inhibiting c-Jun NH2-terminal kinase activation. Biochemical Pharmacology, v. 75, n. 6, p. 1393-1401, mar. 2008. Disponível em: <https://linkinghub.elsevier.com/retrieve/pii/S0006295207007757>.

GUTIÉRREZ-GRIJALVA, E. P. et al. Cellular antioxidant activity and in vitro inhibition of $\alpha$ glucosidase, $\alpha$-amylase and pancreatic lipase of oregano polyphenols under simulated gastrointestinal digestion. Food Research International, v. 116, n. August 2018, p. 676-686, fev. 2019. Disponível em: <https://doi.org/10.1016/j.foodres.2018.08.096>.

HAMEED, S. et al. Synthesis of benzotriazoles derivatives and their dual potential as $\alpha$-amylase and $\alpha$-glucosidase inhibitors in vitro: Structure-activity relationship, molecular docking, and kinetic studies. European Journal of Medicinal Chemistry, v. 183, p. 111677, dez. 2019. Disponível em: <https://linkinghub.elsevier.com/retrieve/pii/S0223523419308219>

HE, Q.; LV, Y.; YAO, K. Effects of tea polyphenols on the activities of $\alpha$-amylase, pepsin, trypsin and lipase. Food Chemistry, v. 101, n. 3, p. 1178-1182, jan. 2007. Disponível em: <https://linkinghub.elsevier.com/retrieve/pii/S030881460600238X>.

HOMOKI, J. R. et al. Anthocyanin composition, antioxidant efficiency, and $\alpha$-amylase inhibitor activity of different Hungarian sour cherry varieties (Prunus cerasus L.). Food Chemistry, v. 194, p. 222-229, mar. 2016. Disponível em: <http://dx.doi.org/10.1016/j.foodchem.2015.07.130>.

KANG, J. G.; PARK, C. Anti-Obesity Drugs: A Review about Their Effects and Safety. Diabetes \& Metabolism Journal, v. 36, n. 1, p. 13, 2012. Disponível em: <http://edmj.org/journal/view.php?doi=10.4093/dmj.2012.36.1.13>.

KATO, C. G. et al. Inhibition of $\alpha$-Amylases by Condensed and Hydrolysable Tannins: Focus on Kinetics and Hypoglycemic Actions. Enzyme Research, v. 2017, p. 1-12, 14 maio 2017. Disponível em: <https://www.hindawi.com/journals/er/2017/5724902/>.

KAUR, L.; JAYASEKERA, S.; MOUGHAN, P. J. Antioxidant Quality of Tea (Camellia sinensis) as Affected by Environmental Factors. In: Processing and Impact on Antioxidants in Beverages. [s.1.] Elsevier, 2014. p. 121-129.

KERIO, L. C. et al. Characterization of anthocyanins in Kenyan teas: Extraction and identification. Food Chemistry, v. 131, n. 1, p. 31-38, mar. 2012. Disponível em: <http://dx.doi.org/10.1016/j.foodchem.2011.08.005>.

LOCHOCKA, K. et al. Green tea extract decreases starch digestion and absorption from a test meal in humans: a randomized, placebo-controlled crossover study. Scientific Reports, v. 5, n. 1, p. 12015, 30 dez. 2015. Disponível em: <http://dx.doi.org/10.1038/srep12015>

LV, H.-P. et al. Identification of the anthocyanins from the purple leaf coloured tea cultivar Zijuan ( Camellia sinensis var. assamica) and characterization of their antioxidant activities. Journal of Functional Foods, v. 17, p. 449-458, ago. 2015. Disponível em: 
MILLER, G. L. Use of Dinitrosalicylic Acid Reagent for Determination of Reducing Sugar. Analytical Chemistry, v. 31, n. 3, p. 426-428, mar. 1959. Disponível em: <https://pubs.acs.org/doi/abs/10.1021/ac60147a030>.

MUKHERJEE, M. Human digestive and metabolic lipases - a brief review. Journal of Molecular catalysis, v. 22, p. 369-376, 2003.

NAMAL SENANAYAKE, S. P. J. Green tea extract: Chemistry, antioxidant properties and food applications - A review. Journal of Functional Foods, v. 5, n. 4, p. 1529-1541, out. 2013. Disponível em: <http://dx.doi.org/10.1016/j.jff.2013.08.011>.

OLIVEIRA, R. et al. Inhibition of Pancreatic Lipase and Triacylglycerol Intestinal Absorption by a Pinhão Coat (Araucaria angustifolia) Extract Rich in Condensed Tannin. Nutrients, v. 7, n. 7, p. 5601-5614, 9 jul. 2015. Disponível em: <http://www.mdpi.com/2072-6643/7/7/5242>.

SEYEDAN, A. et al. Medicinal Plants and Their Inhibitory Activities against Pancreatic Lipase : A Review. Evidence-Based Complementary and Alternative Medicine, p. 13, 2015.

SINGH, R.; AKHTAR, N.; HAQQI, T. M. Green tea polyphenol epigallocatechi3-gallate: Inflammation and arthritis. Life Sciences, v. 86, n. 25-26, p. 907-918, jun. 2010. Disponível em: <http://dx.doi.org/10.1016/j.lfs.2010.04.013>.

SOSNOWSKA, D. et al. Effects of Fruit Extracts on Pancreatic Lipase Activity in Lipid Emulsions. Plant Foods for Human Nutrition, v. 70, n. 3, p. 344-350, 23 set. 2015. Disponível em: <http://link.springer.com/10.1007/s11130-015-0501-x>.

TRINH, B. T. D.; STAERK, D.; JÄGER, A. K. Screening for potential $\alpha$-glucosidase and $\alpha$ amylase inhibitory constituents from selected Vietnamese plants used to treat type 2 diabetes. Journal of Ethnopharmacology, v. 186, p. 189-195, jun. 2016. Disponível em: <http://dx.doi.org/10.1016/j.jep.2016.03.060>.

VIJAYARAJ, P.; NAKAGAWA, H.; YAMAKI, K. Cyanidin and cyanidin - 3 - glucoside derived from Vigna unguiculata act as noncompetitive inhibitors of pancreatic lipase. Journal of food biochemistry, n. October 2018, p. 1-10, 2019.

XU, Y. et al. Seperation, characterization and inhibition on $\alpha$-glucosidase, $\alpha$-amylase and glycation of a polysaccharide from blackcurrant fruits. LWT, v. 93, n. September 2017, p. 1623, jul. 2018. Disponível em: 〈https://doi.org/10.1016/j.lwt.2018.03.023>.

YANG, C. S.; WANG, H.; SHERIDAN, Z. P. Studies on prevention of obesity, metabolic syndrome, diabetes, cardiovascular diseases and cancer by tea. Journal of Food and Drug Analysis, v. 26, n. 1, p. 1-13, jan. 2018. Disponível em: $<$ https://doi.org/10.1016/j.jfda.2017.10.010>. 